\title{
Linx
}

Revue des linguistes de l'université Paris X Nanterre

$57 \mid 2007$

Études de syntaxe : français parlé, français hors de France, créoles

\section{Karima ZIAMARI, Le code switching au Maroc : l'arabe marocain au contact du français}

Paris, L'Harmattan, Col, Espaces discursifs, 2008. Préface de Carol MyersScotton

Driss Ablali

\section{OpenEdition}

\section{Journals}

Édition électronique

URL : http://journals.openedition.org/linx/310

DOI : $10.4000 / \operatorname{linx} .310$

ISSN : 2118-9692

Éditeur

Presses universitaires de Paris Nanterre

Édition imprimée

Date de publication : 1 décembre 2007

Pagination : 177-179

ISSN : 0246-8743

Référence électronique

Driss Ablali, "Karima ZIAMARI, Le code switching au Maroc : l'arabe marocain au contact du français », Linx [En ligne], 57 | 2007, mis en ligne le 15 février 2011, consulté le 22 septembre 2020. URL : http:// journals.openedition.org/linx/310; DOI : https://doi.org/10.4000/linx.310

Ce document a été généré automatiquement le 22 septembre 2020.

Département de Sciences du langage, Université Paris Ouest 


\section{Karima ZIAMARI, Le code switching au Maroc : l'arabe marocain au contact du français}

Paris, L'Harmattan, Col, Espaces discursifs, 2008. Préface de Carol MyersScotton

Driss Ablali

\section{RÉFÉRENCE}

Karima ZIAMARI, Le code switching au Maroc : l'arabe marocain au contact du français, Paris, L’Harmattan, Col, Espaces discursifs, 2008. Préface de Carol Myers-Scotton.

1 Les travaux en sociolinguistique au Maroc constituent l'axe le plus dynamique de la recherche universitaire, et pourtant aucun livre n'est consacré entièrement à la question du code switching. C'est désormais un vide comblé, grâce à l'opus que nous livre Karima Ziamari, Le code switching au Maroc: l'arabe marocain au contact du français. L'ouvrage est consacré à l'analyse linguistique du code switching (alternance codique) entre l'arabe marocain et le français dans le cadre du modèle insertionnel : le Matrix language frame de C. Myers-Scotton, qui préface elle-même ce livre.

L'ouvrage de Karima Ziamari (désormais KZ) est le fruit de sa thèse, presque intouchée, pour le doctorat soutenue en 2003 en cotutelle, INALCO \& Université de Fès, sous la direction de Dominique Caubet et Miloud Taifi. L'étude est basée sur un corpus équivalent à onze heures de conversation enregistré auprès d'étudiants scientifiques marocains (ENSAM, entre autres). Le livre comporte neuf chapitres, dont voici la présentation.

3 Le premier chapitre, «Les modèles linguistiques », présente une revue de la littérature sur le code switching arabe marocain/français depuis le travail d'Abbassi (1977), en passant par les travaux d'A Bentahila \& E. Davies (1983) et d'autres (M. Lahlou 1991) (une période de 20 ans). La première conclusion à laquelle est parvenue cette synthèse 
est l'inadéquation de l'approche linéaire et du modèle du gouvernement pour la description et l'analyse du code switching arabe marocain-français. Les deux modèles dévoilent plusieurs limites et contre-exemples qui les rendent inapplicables.

Dans le deuxième chapitre, «L'arabe marocain et le français en contact : de l'emprunt au code switching ", il s'agit de définir le code switching, de présenter sa typologie, et surtout de le distinguer de l'emprunt linguistique. Ce chapitre, en présentant une typologie du code switching (interphrastique et intraphrastique), précise celui qui a été retenu pour l'analyse du corpus, le code switching intraphrastique. Quant à la différence entre le code switching et l'emprunt linguistique, KZ donne des arguments qui touchent les questions du nombre de mots et le phénomène du lexique mental.

5 Le troisième chapitre, comme l'indique son titre, "Corpus et informateurs ", introduit les deux composantes essentielles de cette recherche. Le corpus équivaut approximativement à onze heures d'enregistrement auprès de trente-trois informateurs (y compris l'enquêtrice) dans deux contextes sociolinguistiques différents: l'espace classe et les espaces moins formels: (cybercafés, résidences universitaires ou compartiments de train...). Ce chapitre expose également la méthodologie adoptée pour la collecte et la présentation des données. Il se termine sur une analyse micro-sociolinguistique des informateurs, affinée grâce à un questionnaire confirmant certaines données relatives à leurs attitudes par rapport aux deux langues : français et arabe marocain, sans oublier l'anglais et l'arabe classique.

6 C'est dans le quatrième chapitre, «L'insertion du français : les constituants mixtes ", que l'on entre dans le vif du sujet: un travail rigoureuxsur l'analyse syntaxique et morphologique des constituants mixtes quand l'arabe marocain est la langue matrice. L'auteure examine aussi les occurrences du code switching arabe marocain/ français, quand la première langue domine, et compare les exemples de son corpus à d'autres paires de langue: arabe marocain/néerlandais, arabe algérien/ français, arabe marocain/espagnol. Ce qui nous donne une idée sur la spécificité de la paire de langues étudiée.

7 Le cinquième chapitre, "L'activation du français, langue enchâssée : les îlots ", décrit les îlots en langue enchâssée et les îlots internes, et avance des explications à ce phénomène tout en comparant le couple arabe marocain/français à d'autres paires de langues. Les îlots en langue enchâssée se présentent sous forme de syntagmes nominaux, de syntagmes prépositionnels et de syntagmes flexionnels. A cet égard, on pourra souligner qu'aucune étude sur cette paire de langue n'a réservé autant d'importance à cette construction.

8 Le sixième chapitre, "L'insertion de l'arabe marocain: l'asymétrie dans le code switching ", s'attèle à l'autre axe du code switching, à savoir l'insertion de l'arabe marocain, en révélant certes la présence des trois structures (les constituants mixtes, les îlots et les îlots internes), mais aussi leur rareté. L'analyse du corpus montre clairement que les deux langues, non seulement ne possèdent pas la même fonction, mais surtout ne jouent pas le même rôle quand elles sont langues matrices. L'insertion du français, contrairement à l'insertion de l'arabe marocain, est quantitativement et qualitativement plus importante.

9 «Le Matrix Language Frame : un modèle valable pour un corpus spécifique ?», comme titre du septième chapitre, insiste sur la critique du modèle à travers trois axes fondamentaux : l'hypothèse de la langue matrice, la congruence et l'introduction des éléments phonologique et énonciatif dans l'analyse. KZ y discute du premier axe: 
l'hypothèse de la langue matrice est vérifiée à travers l'ordre et les types de morphèmes. La question de l'ordre des morphèmes révèle la caractéristique de l'arabe marocain, cette langue matrice qui permet à la fois l'ordre SVO et VSO. Dans les constituants mixtes, l'ordre le plus fréquent est l'ordre partagé par les deux langues: SVO. Cependant, on constate aussi l'ordre VSO dans le corpus. Parmi les résultats qui appuient les hypothèses du Matrix Language Frame demeure l'ordre VSO, observé quand il s'agit des îlots. Une des questions débattues aussi dans ce chapitre est l'accord en tant que manifestation des morphèmes de système.

L'avant dernier chapitre, «La congruence ou la force explicative du MLF », s'attarde sur l'une des notions les plus fondamentales pour les approches du code switching, à savoir la congruence ou la compatibilité entre les deux langues. L'étude conclut que l'arabe marocain et le français diffèrent au niveau de la détermination: le déterminant est certes de type "early system morpheme » dans les deux langues et obéit à la même structure uniforme, mais ses traits restent marqués par la langue dans laquelle il est inséré. En français l'article varie en définitude, genre et nombre, contrairement à l'arabe marocain qui le spécifie pour la définitude uniquement. Ce qui semble expliquer la fréquence des îlots enchâssés, des îlots internes et surtout la rareté des exemples où le déterminant arabe garde sa forme.

11 «La dimension phonologique et énonciative : la limite du Matrix Language Frame » est le dernier chapitre de ce livre. L'auteure y concilie le Matrix Language Frame avec deux niveaux d'analyse : le phonologique et l'énonciatif. Phénomènes rarement exploités en code switching, les dimensions phonétique, phonologique et prosodique n'ont pas été prises en compte par le Matrix Language Frame, sauf s'il s'agit de trancher dans le fameux débat sur la distinction entre code switching et emprunt (l'intégration). Or, l'étude a démontré sur des exemples attestés que l'élément intonatif permet d'identifier la langue matrice. La dimension phonologique est traitée à travers le roulement du R, l'assimilation, le changement dans le point d'articulation.

Le livre de $\mathrm{KZ}$ met fort bien en lumière la validité du cadre conceptuel utilisé, ses qualités et ses lacunes, identifiées comme relevant de la phonologie et des particules énonciatives. S'il est montré que le « Matrix Language Frame » malgré une orientation purement morpho-syntaxique, peut sans difficulté majeure absorber le domaine phonologique en tant qu'indice supplémentaire pour déterminer la langue matrice, celui des particules énonciatives s'avère beaucoup plus problématique. $\mathrm{KZ}$ a certes le mérite de soulever et de cerner rigoureusement le problème, il n'en demeure pas moins qu'aucune solution ne semble s'esquisser pour intégrer la dimension énonciative dans cette théorie.

On pourrait ajouter, pour terminer, que ce livre demeure la meilleure introduction, dans la langue de Molière, aux travaux de Carol Myers-Scotton, mais une introduction, il faut bien le rappeler, appliquée loin des sentiers battus. C'est un travail de randonneur qui écarte la flânerie comme l'aventure pour proposer une marche par étapes, autrement dit une entreprise sérieuse, supposant lecture de cartes et repérages de balises, ce qui n'exclut pas le plaisir de la découverte: des synthèses scandent régulièrement le raisonnement qui se veut accessible à un public élargi. 


\section{AUTEURS}

DRISS ABLALI

LASELDI \& Université de Franche-Comté. 